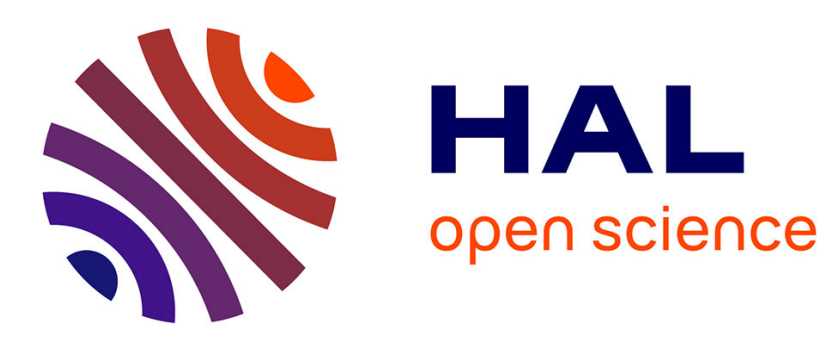

\title{
Autocalibration in the Presence of Critical Motions
}

\author{
David Demirdjian, Gabriela Csurka, Radu Horaud
}

\section{To cite this version:}

David Demirdjian, Gabriela Csurka, Radu Horaud. Autocalibration in the Presence of Critical Motions. 9th British Machine Vision Conference (BMVC '98), Sep 1998, Southampton, United Kingdom. pp.751-759. inria-00590093

\section{HAL Id: inria-00590093 \\ https://hal.inria.fr/inria-00590093}

Submitted on 3 May 2011

HAL is a multi-disciplinary open access archive for the deposit and dissemination of scientific research documents, whether they are published or not. The documents may come from teaching and research institutions in France or abroad, or from public or private research centers.
L'archive ouverte pluridisciplinaire HAL, est destinée au dépôt et à la diffusion de documents scientifiques de niveau recherche, publiés ou non, émanant des établissements d'enseignement et de recherche français ou étrangers, des laboratoires publics ou privés. 


\title{
Autocalibration in the presence of critical motions
}

\author{
David Demirdjian Gabriella Csurka Radu Horaud \\ first. lasteimag. fr \\ GRAVIR-IMAG \& INRIA Rhône-Alpes \\ 655, avenue de l'Europe \\ 38330 Montbonnot Saint Martin, FRANCE
}

\begin{abstract}
Autocalibration is a difficult problem. Not only is its computation very noisesensitive, but there also exist many critical motions that prevent the estimation of some of the camera parameters. When a "stratified" approach is considered, affine and Euclidean calibration are computed in separate steps and it is possible to see that a part of these ambiguities occur during affine-toEuclidean calibration.

This paper studies the affine-to-Euclidean step in detail using the real Jordan decomposition of the infinite homography. It gives a new way to compute the autocalibration and analyzes the effects of critical motions on the computation of internal parameters. Finally, it shows that in some cases, it is possible to obtain complete calibration in the presence of critical motions.
\end{abstract}

Keywords : Autocalibration, critical motions, real Jordan decomposition, affine calibration, infinite homography.

\section{Introduction}

This article raises the problem of autocalibration of a camera undergoing rigid motions under the assumption of constant intrinsic camera parameters.

Many methods of autocalibrating monocular and stereo sensors have been developed in the recent years. Faugeras, Luong and Maybank [FLM92] propose to solve the Kruppa equations from points matches in 3 images. However, this requires non-linear resolution methods. An alternative solution consists to first recover affine structure and then solve for the camera calibration using this. This "stratified" approach [Fau95] can be applied to a single camera motion [LV93] or to a stereo rig in motion [DF96] and requires no knowledge about the observed scene.

Affine calibration has already been studied by many authors and amounts to recovering the equation of the plane at infinity, or equivalently the infinite homographies between the views. Many classes of motions have been treated and theoretically solved : pure translation [RCH98], rotations around the camera's center of projection [Har94], planar motions [BZ95] [CDRH98] and general displacements [ZBR95] [HC98].

The infinite homographies then allow the Euclidean calibration to be computed and it is well known that this computation is possible when at least 2 motions with non zero 
rotations and non parallel rotation axes are available. However, it is not always possible to have such motions. One solution is to add a constraint on the internal parameters (e.g. that the image axes are perfectly orthogonal or that the aspect ratio is known). But, even in this case there exist some critical motions [Stu97] which prevent an unambiguous calibration.

The main contribution of this article is a detailed analysis of affine-to-Euclidean autocalibration, based on the real Jordan decomposition of the infinite homographies. This provides a new way to calculate the Euclidean calibration. Critical motions (where the intrinsic parameters can not all be recovered) are also studied. However, in some cases, if the correct constraint is applied, the problem can be solved and all of the intrinsic parameters can be calculated.

\section{Preliminaries}

A pinhole camera projects a point $\boldsymbol{M}$ from the 3-D projective space onto a point $\boldsymbol{m}$ of the 2 -D projective plane. This projection can be written as a $3 \times 4$ homogeneous matrix $\mathbf{P}$ of rank equal to 3 :

$$
\boldsymbol{m} \simeq \mathbf{P} M
$$

where $\simeq$ is the equality up to a scale factor. If we restrict the 3 -D projective space to the Euclidean space, then it is well known that $\mathbf{P}$ can be written as :

$$
\mathbf{P}=(\mathbf{K R ~ K t})
$$

$\mathbf{R}$ and $\boldsymbol{t}$ are the rotation and translation that link the camera frame to the 3-D Euclidean one. The most general form for the matrix of intrinsic parameters $\mathbf{K}$ is :

$$
\left(\begin{array}{ccc}
\alpha & r \alpha & u_{0} \\
0 & k \alpha & v_{0} \\
0 & 0 & 1
\end{array}\right)
$$

where $\alpha$ is the horizontal scale factor, $k$ is the ratio between the vertical and horizontal scale factors, $r$ is the image skew and $u_{0}$ and $v_{0}$ are the image coordinates of the center of projection.

\section{From affine to Euclidean}

The affine calibration enables to calculate the infinite homography $\mathbf{H}_{\infty}$ between the images taken with a camera to calibrate, before and after the rigid motion. Once this infinite homography is obtained, it is possible to recover $\mathbf{K}$ thanks to the relation :

$$
\mathbf{H}_{\infty}=\mathbf{K R K}^{-1}
$$

where $\mathbf{R}$ is the rotation of the motion (the Euclidean frame is chosen to be the camera frame). A classical way to solve this equation was first proposed by R.Hartley [Har94] and consists to solve the equation : 


$$
\mathbf{H}_{\infty}^{T} \mathbf{C H}_{\infty}=\mathbf{C}
$$

where $\mathbf{C}=\mathbf{K}^{-T} \mathbf{K}^{-1}$ is the image of the absolute conic. $\mathbf{K}$ is then obtained by Cholesky decomposition of $\mathbf{C}$. It is well known that the solutions $\mathbf{C}$ of (2) define a 1parameter family. This ambiguity can be eliminated if at least 2 motions with non parallel rotation axes are considered.

We propose another way to solve (1) with an analysis which allows us to identify critical motions and to partially solve for calibration in these particular cases. This analysis is based on the real Jordan decomposition which has already been studied in [CDRH98] in the case of projective displacements. The approach takes into account the fact that (1) defines $\mathbf{H}_{\infty}$ as the conjugate of a rotation $\mathbf{R}$. In an appropriate frame, $\mathbf{R}$ can be reduced to the simple form $\mathbf{J}_{\theta}$ :

$$
\mathbf{J}_{\theta}=\left(\begin{array}{ccc}
\cos (\theta) & -\sin (\theta) & 0 \\
\sin (\theta) & \cos (\theta) & 0 \\
0 & 0 & 1
\end{array}\right)
$$

Then, there exists a $3 \times 3$ matrix $\mathbf{S}$ such that :

$$
\mathbf{H}_{\infty}=\mathbf{S J}_{\theta} \mathbf{S}^{-1}
$$

This is a real Jordan decomposition of $\mathbf{H}_{\infty}$.

\subsection{Analysis of the real Jordan decomposition}

\subsubsection{Ambiguity}

In (3), $\theta$ is uniquely determined. However, $\mathbf{S}$ is not. Indeed, if $\mathbf{S}$ satisfies (3) and $\mathbf{P}$ is any invertible matrix which commutes with $\mathbf{J}_{\theta}$, we have :

$$
\begin{aligned}
\mathbf{H}_{\infty} & =\mathbf{S} \mathbf{J}_{\theta} \mathbf{P} \mathbf{P}^{-1} \mathbf{S}^{-1} \\
& =\mathbf{S P J}_{\theta} \mathbf{P}^{-1} \mathbf{S}^{-1} \\
& =(\mathbf{S P}) \mathbf{J}_{\theta}(\mathbf{S P})^{-1}
\end{aligned}
$$

So, $\mathbf{S}^{\prime}=\mathbf{S P}$ satisfies (3) too. The converse is also true. That is, if $\mathbf{S}_{1}$ and $\mathbf{S}_{2}$ both satisfy (3), then $\mathbf{S}_{2}^{-1} \mathbf{S}_{1}$ is a matrix which commutes with $\mathbf{J}_{\theta}$. It can be easily shown that such a matrix can be written :

$$
\mathbf{P}_{a, b, c}=\left(\begin{array}{ccc}
a & -b & 0 \\
b & a & 0 \\
0 & 0 & c
\end{array}\right)
$$

Let $\mathbf{Q}^{1}$ be an orthogonal matrix such that $\mathbf{R}=\mathbf{Q} \mathbf{J}_{\theta} \mathbf{Q}^{T}$. (1) gives a real Jordan decomposition for $\mathbf{H}_{\infty}$ :

$$
\mathbf{H}_{\infty}=\mathbf{K Q J}_{\theta}(\mathbf{K Q})^{-1}
$$

\footnotetext{
${ }^{1} \mathbf{Q}$ is the transformation which enables to express the rotation in a canonic frame where the rotation axis is the $z$-axis.
} 
Furthermore, if $\mathbf{S}$ has been calculated by a real Jordan decomposition of $\mathbf{H}_{\infty}$ (we will see later how to obtain it), we have, with respect to what has been shown previously, the following relationship :

$$
\mathbf{S P}_{a, b, c}=\mathbf{K Q}
$$

Then,

$$
(\mathbf{K Q})(\mathbf{K Q})^{T}=\left(\mathbf{S P}_{a, b, c}\right)\left(\mathbf{S P}_{a, b, c}\right)^{T}
$$

And so, by $\mathbf{Q} \mathbf{Q}^{T}=\mathbf{I}$,

$$
\mathbf{K K}^{T}=\mathbf{S}\left(\begin{array}{ccc}
a^{2}+b^{2} & 0 & 0 \\
0 & a^{2}+b^{2} & 0 \\
0 & 0 & c^{2}
\end{array}\right) \mathbf{S}^{T}
$$

Let be $\lambda=a^{2}+b^{2}$ and $\nu=c^{2}$. We have finally:

$$
\mathbf{K K}^{T}=\mathbf{S}\left(\begin{array}{ccc}
\lambda & 0 & 0 \\
0 & \lambda & 0 \\
0 & 0 & \nu
\end{array}\right) \mathbf{S}^{T}
$$

\subsubsection{Particular forms of $\mathrm{S}$}

For any motion, the relation (4) implies that the form of $\mathbf{S}$ is :

$$
\mathbf{S}=\mathbf{K Q P}_{a, b, c}^{-1}=\mathbf{K Q P} \mathbf{P}_{a^{\prime}, b^{\prime}, c^{\prime}}
$$

where $\mathbf{P}_{a^{\prime}, b^{\prime}, c^{\prime}}=\mathbf{P}_{a, b, c}^{-1}$ commutes with $\mathbf{J}_{\theta}$.

When the rotation component of the displacement is performed around an axis parallel to the basis axes of the camera, $\mathbf{S}$ takes special forms :

- If the rotation axis is parallel to the horizontal axis of the camera :

$$
\mathbf{Q}=\left(\begin{array}{lll}
0 & 0 & 1 \\
1 & 0 & 0 \\
0 & 1 & 0
\end{array}\right) \quad \text { and } \quad \mathbf{S} \simeq \mathbf{S}_{x} \simeq\left(\begin{array}{ccc}
* & * & 1 \\
* & * & 0 \\
* & * & 0
\end{array}\right)
$$

- If the rotation axis is parallel to the vertical axis of the camera :

$$
\mathbf{Q}=\left(\begin{array}{ccc}
0 & 1 & 0 \\
0 & 0 & 1 \\
1 & 0 & 0
\end{array}\right) \quad \text { and } \quad \mathbf{S} \simeq\left(\begin{array}{ccc}
* & * & \frac{r}{k} \\
* & * & 1 \\
* & * & 0
\end{array}\right)
$$

In practice, $r$ is often negligible in comparison with $k$ and we can consider that :

$$
\mathbf{S} \simeq \mathbf{S}_{y} \simeq\left(\begin{array}{ccc}
* & * & 0 \\
* & * & 1 \\
* & * & 0
\end{array}\right)
$$


- Finally, if the rotation axis is orthogonal to the image plane, $\mathbf{Q}$ is the identity and :

$$
\mathbf{S} \simeq \mathbf{S}_{z} \simeq\left(\begin{array}{ccc}
* & * & u_{0} \\
* & * & v_{0} \\
0 & 0 & 1
\end{array}\right)
$$

We can observe that, in these 3 cases, the structure of $\mathbf{S}$ is independent of any ambiguity in the real Jordan decomposition. It will be shown later that these cases correspond to critical motions for affine-to-Euclidean calibration.

\subsection{Affine-to-Euclidean calibration}

The real Jordan decomposition can easily be obtained from the eigenvectors of $\mathbf{H}_{\infty}$.

Indeed, let $\left\{e^{i \theta}, e^{-i \theta}, 1\right\}$ be the eigenvalues of $\mathbf{H}_{\infty}^{2}$ and $\boldsymbol{u}_{1}, \boldsymbol{u}_{2}=\overline{\boldsymbol{u}_{1}}$ and $\boldsymbol{u}_{\mathbf{3}}$ be the associated eigenvectors.

If $\boldsymbol{v}_{\mathbf{1}}=\boldsymbol{u}_{\mathbf{1}}+\boldsymbol{u}_{\mathbf{2}}, \boldsymbol{v}_{\mathbf{2}}=i\left(\boldsymbol{u}_{\mathbf{1}}-\boldsymbol{u}_{\mathbf{2}}\right)$ and $\mathbf{S}=\left(\boldsymbol{v}_{\mathbf{1}} \boldsymbol{v}_{\mathbf{2}} \boldsymbol{u}_{\mathbf{3}}\right)$, then we have $\mathbf{H}_{\infty}=\mathbf{S} \mathbf{J}_{\theta} \mathbf{S}^{-1}$.

\subsubsection{Resolution of (5)}

As we have seen previously, given an infinite homography $\mathbf{H}_{\infty}$ between the images of the same camera, it is possible to calculate its real Jordan decomposition and hence a matrix $\mathbf{S}$ such that $\mathbf{H}_{\infty}=\mathbf{S} \mathbf{J}_{\theta} \mathbf{S}^{-1}$. Then, it was shown that $\mathbf{S}$ should satisfy (5). The calibration process consists of solving this equation which can be written as a system of 6 equations with 7 unknowns $\left(a, k, u_{0}, v_{0}, r, \lambda\right.$ and $\left.\nu\right)$ :

$$
\begin{aligned}
& \alpha^{2}+\alpha^{2} r^{2}+u_{0}^{2}-S_{1,1}^{2} \lambda-S_{1,2}^{2} \lambda-S_{1,3}^{2} \nu=0 \\
& k^{2} \alpha^{2}+v_{0}^{2}-S_{2,1}{ }^{2} \lambda-S_{2,2}^{2} \lambda-S_{2,3}^{2} \nu=0 \\
& 1-S_{3,1}{ }^{2} \lambda-S_{3,2}{ }^{2} \lambda-S_{3,3}{ }^{2} \nu=0 \\
& r k \alpha^{2}+u_{0} v_{0}-S_{1,1} \lambda S_{2,1}-S_{1,2} \lambda S_{2,2}-S_{1,3} \nu S_{2,3}=0 \\
& u_{0}-S_{1,1} \lambda S_{3,1}-S_{1,2} \lambda S_{3,2}-S_{1,3} \nu S_{3,3}=0 \\
& v_{0}-S_{2,1} \lambda S_{3,1}-S_{2,2} \lambda S_{3,2}-S_{2,3} \nu S_{3,3}=0
\end{aligned}
$$

It is clear that this system cannot be solved just as it is (there is one unknown too many). We must either consider a constraint on one unknown, or add equations from several motions (with non parallel rotation axes).

If we want to calibrate with a single motion or with planar motions (where all rotation axes are parallel), a constraint on the internal parameters must be imposed. Commonly used constraints are either $k=k_{0}$ or $r=0$.

\section{General resolution}

If the matrix $\mathbf{S}$ does not have one of the forms expressed in 3.1.2, the system can then be solved:

- if the constraint $r=0$ is used, the intrinsic parameters are uniquely defined.

\footnotetext{
${ }^{2}$ We suppose that $\mathbf{H}_{\infty}$ is normalized such that its determinant is equal to 1.0. Since it is conjugate to a rotation matrix, it has one real and one complex conjugate pair of eigenvalues, all of unit modulus.
} 


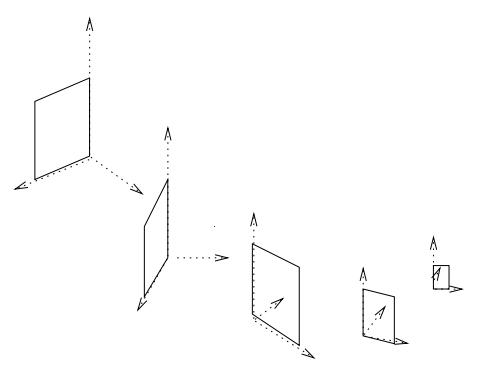

Figure 1: example of critical motion : vertical rotation axis

- if the constraint $k=k_{0}$ is chosen, there are 2 sets of solutions : they correspond to the 2 solutions $\lambda$ of a second degree equation. We keep only the one with smallest $|r|$.

Now consider the degenerate cases.

\section{Degenerate cases}

- Horizontal rotation axis. We have $\mathbf{S} \simeq \mathbf{S}_{x}$ and so, $S_{3,3}=0$ and $S_{2,3}=0$ (see (6)) : (11) gives $\lambda$ and $u_{0}$ and $v_{0}$ can then be computed with (13) and (14).

- if we impose $r=0,(10)$ gives $k \alpha$. However, (9) can't give either $\alpha$ or $\nu$.

- if $k=k_{0}$ is considered, $\alpha^{2}+\alpha^{2} r^{2}$ and $\alpha^{2} r$ can be calculated directly and solved to give $r, \alpha_{u}=\alpha$ and $\alpha_{v}=k \alpha$.

- Vertical rotation axis. This is the case when $\mathbf{S} \simeq \mathbf{S}_{y}$, which is similar to the previous one. We have $S_{3,3}=0$.

- if $r=0$, we have $S_{1,3}=0$ and then all parameters except $k$ and $k \alpha$ can be evaluated.

- if $k=k_{0}, S_{1,3}=\frac{r}{k}$ leads to a total resolution of calibration

- Rotation axis orthogonal to the image plane. We have $\mathbf{S} \simeq \mathbf{S}_{z}\left(S_{3,1}=0\right.$ and $S_{3,2}=0$ ).

It is the worst critical case in so far as $u_{0}, v_{0}, k$ and $r$ can be calculated, but $\alpha$ remains always undetermined, whatever the constraint may be.

As a conclusion, we saw that the problem of affine-to-Euclidean calibration could be easily solved in particular cases (single motion, all parallel axes rotations ${ }^{3}$ ).

We also showed that using the constraint $k=k_{0}$ allowed us to avoid critical cases : there remains then just one real critical motion (rotation axes orthogonal to the image plane).

\footnotetext{
${ }^{3}$ In this case, it is possible to calculate a matrix $\mathbf{S}$ that satisfi es the real Jordan decomposition of each infi nite homography.
} 


\section{Experiments and results}

\subsection{Description}

In this section we apply our autocalibration algorithms to synthetic data in order to analyse the effect of different kind of motions on the computation of autocalibration. 3-D points were generated and projected onto the cameras of a virtual stereo rig $^{4}$ performing different kind of motions. Gaussian noise of 1-pixel standard deviation was added to the data.

For simplicity, we show results only for the calibration of the left camera of the rig. The actual intrinsic parameters are :

$$
\mathbf{K}=\left(\begin{array}{ccc}
715 & 0 & 140 \\
0 & 995 & 275 \\
0 & 0 & 1
\end{array}\right)
$$

The aim of this experiment is not to obtain accurate computation of intrinsic parameters, but to show that if the constraint $k=k_{0}$ is used, there is only one critical kind of motions for the affine-to-Euclidean calibration (instead of three) : motions whose rotation axes are orthogonal to the image plane.

\subsection{Results}

First, projective displacements $D_{p r o j}$ are calculated from point correspondences and epipolar geometry with the method described in [HC98]. Then, the equation of the plane at infinity is calculated and the infinite homographies $\mathbf{H}_{\infty}$ associated to the left camera are derived. Our affine calibration algorithms are similar to [BZ95] and [HC98] and cope with general and planar motions (in this case, we need at least 2 motions). Finally, the real Jordan decomposition of each $\mathbf{H}_{\infty}$ is calculated and the resolution of (5) enables us to obtain the complete camera calibration.

We show the results on 4 motion sequences (each consisting of 5 motions) :

- sequence 1 : non singular general motions

- sequence 2 : non singular planar motions

- sequence 3 : planar motion with a horizontal rotation axis

- sequence 4 : planar motion with a vertical rotation axis

The following table exhibits the matrices $\mathbf{H}_{\infty}$ and $\mathbf{S}$ obtained for the first motion of each sequence. It confirms the particular forms of $\mathbf{S}$ obtained for critical motions.

\footnotetext{
${ }^{4}$ Intrinsic parameters of each camera were constant.
} 


\begin{tabular}{|c|c|c|c|c|c|c|}
\hline & \multicolumn{3}{|c|}{$\mathbf{G}_{\infty}$} & \multicolumn{3}{|c|}{$\mathbf{S}$} \\
\hline \multirow{3}{*}{ sequence 1} & 0.778 & 0.135 & 315.085 & 0.301 & -0.902 & 1.000 \\
\hline & -0.70 & 0.768 & 354.918 & 1.430 & -0.298 & -1.184 \\
\hline & -0.00 & -0.00 & 1.018 & 0.001 & 0.001 & 0.001 \\
\hline \multirow{3}{*}{ sequence 2} & 1.014 & -0.086 & -161.587 & 2.750 & -0.979 & 1.000 \\
\hline & 0.254 & 1.006 & -129.024 & 0.350 & -2.859 & -3.197 \\
\hline & 0.000 & 0.000 & 0.891 & -0.001 & -0.004 & 0.002 \\
\hline \multirow{3}{*}{ sequence 3} & 1.003 & 0.034 & -8.576 & -0.112 & 0.034 & 1.000 \\
\hline & 0.008 & 1.044 & -297.574 & -0.414 & -0.904 & 0.037 \\
\hline & 0.000 & 0.000 & 0.875 & -0.001 & 0.000 & 0.000 \\
\hline \multirow{3}{*}{ sequence 4} & 1.009 & 0.003 & -214.246 & 0.879 & -0.327 & -0.007 \\
\hline & 0.116 & 1.003 & -26.163 & -0.073 & -0.360 & 1.000 \\
\hline & 0.000 & 0.000 & 0.903 & -0.000 & -0.001 & 0.000 \\
\hline
\end{tabular}

The results of the computation of $\mathbf{K}$ from the previous matrices $\mathbf{S}$ are as follows :

- with the assumption $r=0$, only the first two sequences allow us to calculate $\mathbf{K}$ :

\begin{tabular}{|c|c|c|c|c|c|}
\hline & $\alpha_{u}$ & $\alpha_{v}$ & $k=\frac{\alpha_{v}}{\alpha_{u}}$ & $u_{0}$ & $v_{0}$ \\
\hline sequence 1 & 722.5 & 1002.6 & 1.388 & 126.2 & 280.0 \\
sequence 2 & 695.7 & 939.50 & 1.350 & 147.5 & 273.3 \\
sequence 3 & $\mathbf{8 2 1 . 0}$ & 978.8 & $\mathbf{1 . 1 9 2}$ & 138.05 & 294.18 \\
sequence 4 & 716.43 & $\mathbf{7 0 2 . 2 8}$ & $\mathbf{0 . 9 8 0}$ & 135.05 & 275.62 \\
\hline
\end{tabular}

- the assumption $k=\frac{995}{715} \approx 1.39$ allows us to calculate $\mathbf{K}$ in all sequences :

\begin{tabular}{|l|c|c|c|c|c|}
\hline & $\alpha_{u}$ & $\alpha_{v}$ & $r$ & $u_{0}$ & $v_{0}$ \\
\hline sequence 1 & 721.8 & 1007.8 & -0.0164 & 139.7 & 255.4 \\
sequence 2 & 691.8 & 966.0 & -0.0137 & 153.9 & 241.0 \\
sequence 3 & 700.8 & 978.6 & -0.0272 & 117.6 & 293.4 \\
sequence 4 & 712.5 & 994.5 & 0.0046 & 127.8 & 283.7 \\
\hline
\end{tabular}

From these experiments, we can see clearly that the $k$-constraint allows us to calibrate even in the case of critical motions. With the $r$-constraint, we can see that significant errors are made in $\alpha_{u}$ for sequence 3 , and in $\alpha_{v}$ for sequence 4 .

\section{Conclusion}

We have described a method for solving the problem of affine-to-Euclidean calibration, based on the real Jordan decomposition of $\mathbf{H}_{\infty}$. This allows us to express the ambiguity in the computation of the absolute conic (and also, the intrinsic parameters) as a 1-parameter family. Although this ambiguity can be solved when many motions with non parallel rotation axes are used, it can not when motions are planar or when we dispose of a single motion. In these cases, we showed that an assumption should be made on one of the internal parameters $r$ or $k$. 
Besides, we showed the existence of 3 classes of critical motion. We saw that $r$ and $k$ didn't have the same role in the resolution of the equations : in particular, the knowledge of $k$ allows us to cope with 2 of the 3 critical motions. Experiments on noisy synthetic data confirmed the theoretical results and proved it was possible to calibrate a camera in some of the special cases. Experiments we have made on real data (not shown) seem to confirm that in general resolution of the equations with the $k$-constraint is relatively stable even when the rotation axis is near to the horizontal or vertical axes of the camera.

However, the analysis we made here is only qualitative : we studied which parameters could be obtained for each kind of critical motion. We are currently studying more quantitative extensions to this work which try to analyze the precision and stability of the parameter computation as a function of the motion.

\section{References}

[BZ95] P.A. Beardsley and A. Zisserman. Affine calibration of mobile vehicles. In R. Mohr and C. Wu, editors, Europe-China Workshop on Geometrical Modelling and Invariants for Computer Vision, Xian, China, pages 214-221. Xidan University Press, April 1995.

[CDRH98] G. Csurka, D. Demirdjian, A. Ruf, and R. Horaud. Closed-form solutions for the euclidean calibration of a stereo rig. In Proceedings of the 5th European Conference on Computer Vision, Freiburg, Germany, pages 426-444, 1998.

[DF96] F. Devernay and O. Faugeras. From projective to euclidean reconstruction. In Proceedings of the Conference on Computer Vision and Pattern Recognition, San Francisco, California, USA, pages 264-269, June 1996.

[Fau95] O. Faugeras. Stratification of three-dimensional vision: Projective, affine and metric representations. Journal of the Optical Society of America, 12:465484, 1995 .

[FLM92] O.D. Faugeras, Q.T. Luong, and S.J. Maybank. Camera self-calibration: Theory and experiments. In G. Sandini, editor, Proceedings of the 2nd European Conference on Computer Vision, Santa Margherita Ligure, Italy, pages 321334. Springer-Verlag, May 1992.

[Har94] R.I. Hartley. Self-calibration from multiple views with a rotating camera. In Proceedings of the 3rd European Conference on Computer Vision, Stockholm, Sweden, pages 471-478. Springer-Verlag, May 1994.

[HC98] R. Horaud and G. Csurka. Self-calibration and euclidean reconstruction using motions of a stereo rig. In Proceedings of the 6th International Conference on Computer Vision, Bombay, India, pages 96-103, January 1998.

[LV93] Q.T. Luong and T. Vieville. Canonic representations for the geometries of multiple projective views. Technical report, University of California, Berkeley, EECS, Cory Hall 211-215, University of California, Berkeley, CA 94720, October 1993. 
[RCH98] A. Ruf, G. Csurka, and R. Horaud. Projective translations and affine stereo calibration. In Proceedings IEEE Conference on Computer Vision and Pattern Recognition, pages 475-481, Santa Barbara, CA, June 1998. IEEE Computer Society Press, Los Alamitos, Ca.

[Stu97] P. Sturm. Critical motion sequences for monocular self-calibration and uncalibrated euclidean reconstruction. In Proceedings of the Conference on Computer Vision and Pattern Recognition, Puerto Rico, USA, pages 1100-1105, June 1997.

[ZBR95] A. Zisserman, P.A. Beardsley, and I.D. Reid. Metric calibration of a stereo rig. In Workshop on Representation of Visual Scenes, Cambridge, Massachusetts, USA, pages 93-100, June 1995. 